\title{
On the Accuracy of the Gaussian Nonlinear Model for Dispersion-unmanaged Coherent Links
}

\author{
P. Serena and A. Bononi \\ Università degli Studi di Parma, dept. Ingegneria dell'Informazione, v.le G. P. Usberti 181/A, 43124 \\ Parma (Italy), $\bowtie$ paolo.serena@unipr.it
}

Abstract We discuss the reasons why the Gaussian nonlinear model provides accurate bit error rate predictions in dispersion unmanaged PDM-QPSK coherent links.

\section{Introduction}

The recently discovered quasi-Gaussian nature of the statistics of the nonlinear interference (NLI) in dispersion unmanaged (DU) coherent optical links with single-carrier modulations stimulated the development of several analytical models to evaluate the variance of the $\mathrm{NLI}^{1-3}$, and of elementary bit error rate (BER) formulas based on the received signal to noise ratio $(\mathrm{SNR})^{4,5}$. In this scenario, the Gaussian nonlinear (GN) model ${ }^{1,6,7}$ is particularly attractive, since it is able to provide a simple analytical formula for the NLI power spectral density (PSD). The common assumption shared by all the above models is that nonlinearity is a small perturbation of the nonlinear Schrödinger equation (NLSE). The extra peculiar assumption of the GN model is that the input modulated signal is a Gaussian stochastic pro$\operatorname{cess}^{1,7,8}$.

In this work we wish to add to the debate on the accuracy of the GN model. First, we check the small-perturbation assumption in polarizationdivision multiplexing quadrature phase shift keying (PDM-QPSK) transmissions, showing that it leads to a BER under-estimation. Then, we show that assuming Gaussian input signal statistics over-estimates the true NLI variance, hence the BER. The two errors partially compensate, yielding a good accuracy of the GN model for BER prediction.

\section{Accuracy of small-perturbation assumption}

In this section we quantify the accuracy of the first order perturbation solution of the NLSE, without any assumption on the received NLI statistics. We numerically simulated a $20 \times 100 \mathrm{~km}$ DU link with single mode fibers (SMF) having dispersion 17 $\mathrm{ps} / \mathrm{nm} / \mathrm{km}$ and nonlinear index $\gamma=1.3 \mathrm{~W}^{-1} \mathrm{~km}^{-1}$. At the transmitter we sent 15 PDM-QPSK channels at $R=112 \mathrm{Gbit} / \mathrm{s}$ with $50 \mathrm{GHz}$ spacing. Each channel had 4096 random symbols and random carrier state of polarization (SOP). Fiber propagation was modeled using either the enhanced first

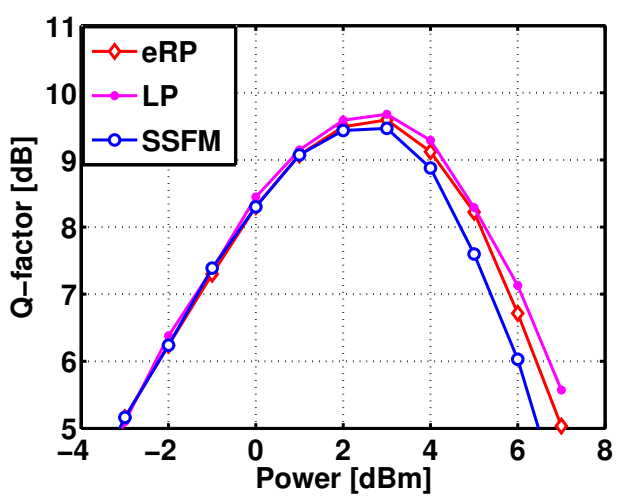

Fig. 1: Monte Carlo estimation of Q-factor using two different perturbation models: eRP and LP. SSFM: split step Fourier method (exact) solution.

order regular perturbation (eRP) ${ }^{9}$ or the logarithmic perturbation (LP) ${ }^{10}$, and solved using the algorithm in ${ }^{9}$. Polarization mode dispersion (PMD) was neglected. The receiver digital signal processor (DSP) performed standard operations, including: ideal compensation of chromatic dispersion; analog to digital conversion with bandwidth $0.6 R$; fractionally spaced 15 taps trained least squares butterfly equalization at 2 samples/symbol. Amplified spontaneous emission (ASE) noise was added at the end of the link with a total noise figure of $25 \mathrm{~dB}$. Performance was estimated in terms of BER through Monte Carlo simulations counting at least 100 errors, and converted to $Q$-factor.

Fig. 1 shows the estimated $Q$-factor vs. power using the two perturbation algorithms. For reference, we also report the split step Fourier method (SSFM) curve, which we will refer to as the true performance. We note that in DU links the LP solution is less accurate than the eRP one, while the opposite is true in dispersion-managed links where the Kerr effect manifests mainly as a phase rotation. In the strongly nonlinear regime (the descending part of Q-factor) the eRP solution overestimates the Q-factor by roughly $1 \mathrm{~dB}$, an error that is reduced near the optimal power by the impact of ASE noise, which is exactly captured by 


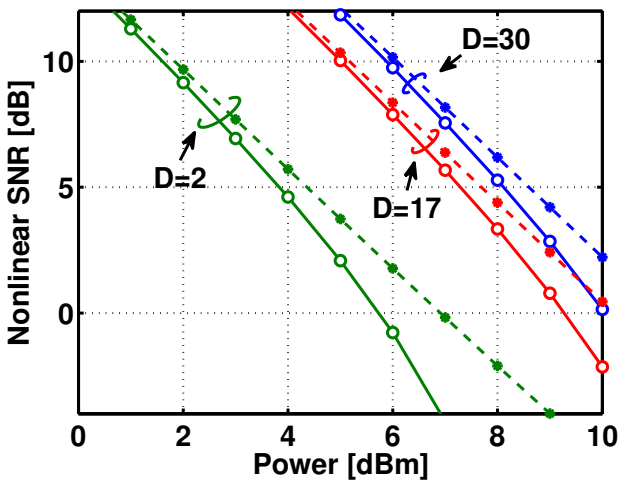

Fig. 2: Monte Carlo estimated SNR in absence of ASE noise for different fiber dispersions $D$ [ps $/ \mathrm{nm} / \mathrm{km}]$. Dashed lines: eRP algorithm. Solid lines: SSFM.

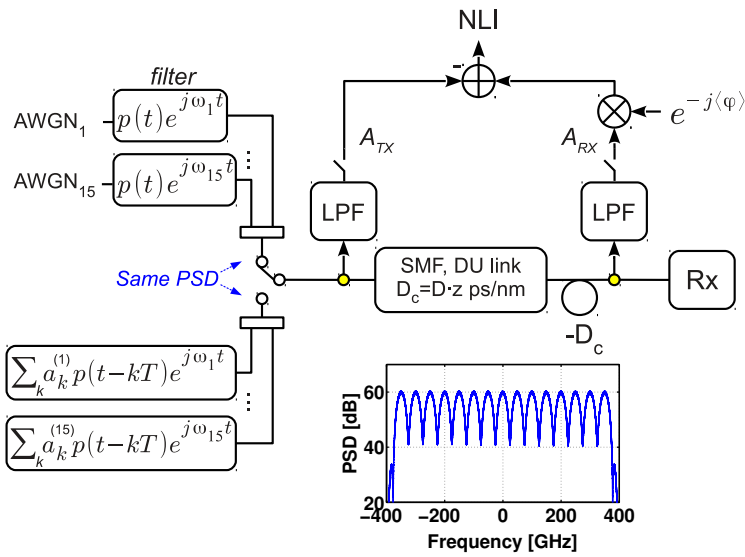

Fig. 3: Test for the Gaussian assumption. The signal entering the $z=20 \times 100 \mathrm{~km}$ link is either PDM-QPSK or AWGN with identical PSD after filtering.

all models.

To quantify the impact of fiber dispersion $D$, we measured the SNR of the received constellation clouds in absence of ASE noise, both with the eRP and the SSFM algorithms. Such an SNR is reported in Fig. 2 with dashed (eRP) and solid lines (SSFM). It is worth noting that at a true nonlinear SNR $>4 \mathrm{~dB}$ the eRP algorithm overestimates the value by less than $1 \mathrm{~dB}$, whatever the fiber dispersion. Such a value of nonlinear SNR can be assumed as a minimum threshold for the applicability of the eRP perturbation model.

\section{Accuracy of input Gaussian assumption}

The previous section showed that the eRP solution of the NLSE over-estimates the Q-factor, and thus the SNR. In this section we show that forcing input Gaussian statistics indeed under-estimates the Q-factor, thereby counteracting the bias of the eRP solution. This claim is supported by the simulation described in Fig. 3. In a noiseless DU link we sent 15 channels, each discretized by $2^{22}$

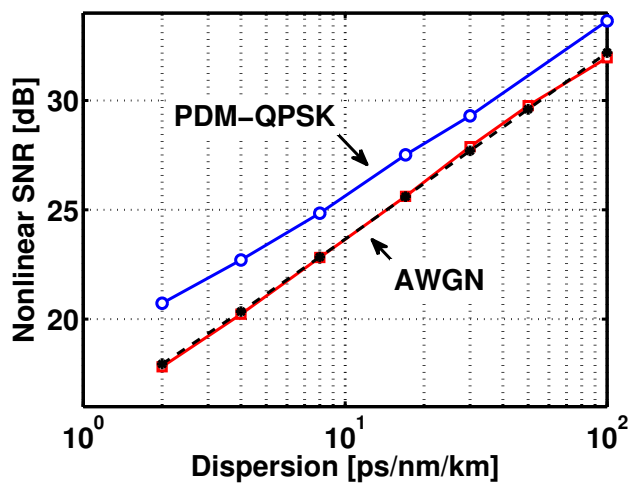

Fig. 4: Nonlinear SNR of link in Fig. 3. Dashed line: Prediction of the GN model.

points and modulated with either a 112 Gbit/s PDM-QPSK signal or with dual-polarization additive white Gaussian noise (AWGN), suitably filtered to yield the same PSD as in Fig. 3. Power was $-4 \mathrm{dBm}$, thus low enough to claim that eRP coincides with SSFM, according to Fig. 2. After the link we extracted the central channel with a 2nd-order super-Gaussian low pass filter (LPF) of bandwidth $25 \mathrm{GHz}$ and then measured the nonlinear SNR by calculating the NLI noise as depicted in Fig. 3, thus as $N L I=A_{R X} \exp (-j\langle\varphi\rangle)-A_{T X}$, being $\langle\varphi\rangle$ the average nonlinear phase rotation induced by the link. The same procedure was used in both the PDM-QPSK and the AWGN cases.

The SNR is reported in Fig. 4 vs. fiber dispersion $D$. Surprisingly, we note that PDM-QPSK is almost $2 \mathrm{~dB}$ above the AWGN. As a sanity check, we numerically solved the double frequency integral of the GN model (eq. (1) of ${ }^{6}$ ) obtaining the dashed black curve that almost perfectly matches with the AWGN Monte-Carlo simulated curve. This fact gives us confidence that also the PDM-QPSK SNR evaluation is correct. In the PDM-QPSK case we also tried to move closer to the assumptions required by the central limit theorem by adding a pre-compensating fiber before transmission (exactly compensated then at the receiver), but the gap with the AWGN case was reduced by only $0.6 \mathrm{~dB}$ at the remarkable pre-dispersion value of $10000 \mathrm{ps} / \mathrm{nm}$.

In a final set of simulations we changed the statistics of the modulating symbols $a_{k}$ in Fig. 3 and rescaled the obtained fields to have the same PSD. We assumed independent in-phase and quadrature symbols drawn from basic distributions like hyperbolic secant, Gaussian, Laplace, logistic and uniform, up to forming a 1024-point constellation. Since the PSD is fixed in all cases 

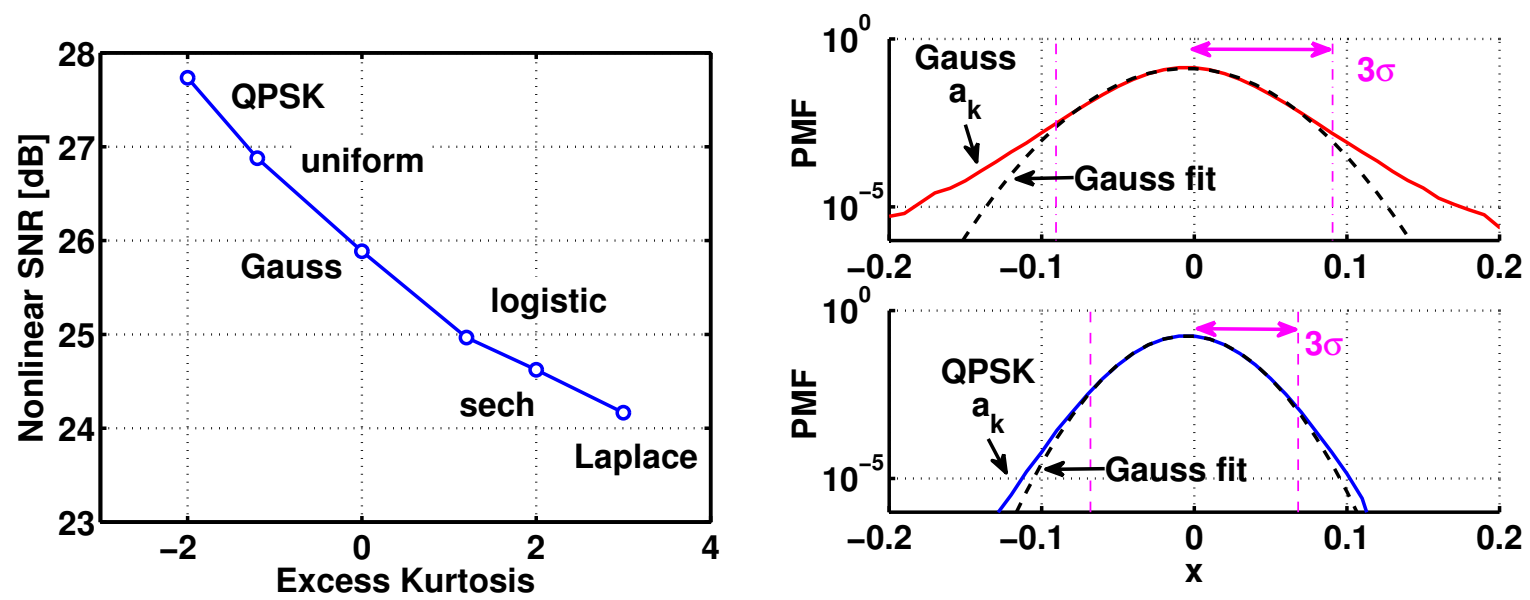

Fig. 5: Left: Nonlinear SNR vs. excess kurtosis $\kappa$ of constellation symbols $a_{k}$ when $D=17 \mathrm{ps} / \mathrm{nm} / \mathrm{km}$. The symbols distribution is indicated in the graph. $\kappa>0$ indicates distributions with heavier tails than Gaussian distribution. Right: PMF of real component of NLI noise assuming QPSK or Gaussian distributed $a_{k}$. PSD is equal for all cases.

by the common supporting pulse spectrum, the difference in behaviors should be searched in higher statistical moments than the variance. Although 6-th order moments of the input field are involved in the analytical GN model computations ${ }^{7,8}$, we found that the fourth moment, expressed by the excess kurtosis $\kappa$, is a good discriminator of the different cases. The excess kurtosis is a measure of the peakedness of a distribution: positive $\kappa$ indicates distributions with heavier tails than the Gaussian one, and vice versa. The information brought by $\kappa$ is similar in spirit to the peak to average power ratio (PAPR), even though it correctly weights the occurrence of rare events like the peak values.

Fig. 5(left) indicates that there is a monotonic relation between the SNR, measured after the DSP, and $\kappa$, despite the large chromatic dispersion accumulated in the link, thereby dispelling the myth that any input signal into a highlydispersive DU link evolves into a Gaussian process. Note that the Gaussian-distributed modulating symbols ("Gauss" label) yield almost the same SNR as the AWGN. In Fig. 5(right) we also reported the probability mass function (PMF) of the real component of the received optical NLI for both the QPSK and Gaussian case. The imaginary component shows an almost identical PMF. It is worth noting that while the SNR, and hence the variance $\sigma^{2}$ of $\mathrm{NLI}$, is distribution-dependent, both PMFs are very well approximated by a Gaussian distribution of variance $\sigma^{2}$ (dashed lines) within a wide range of $\pm 3 \sigma$ from the average value, thus justifying the SNR-based BER formula $^{4,5}$.

\section{Conclusions}

We showed that the GN model for DU links ${ }^{6}$ works because its first-order perturbation assumption over-estimates the $\mathrm{Q}$-factor, while its Gaussian input signal statistics assumption overestimates the nonlinear interference variance, thus partially counterbalancing the first-order perturbation error.

\section{References}

1 A. Carena et al., J. Lightw. Technol. 30, 15241539 (2012).

2 A. Mecozzi et al., J. Lightw. Technol. 30, 20112024 (2012).

3 A. Bononi et al., Opt. Express 20, B204-B216 (2012).

4 G. Bosco et al., in Proc. OFC11, Paper OThO7 (2011).

5 E. Grellier et al., Opt. Express 19, 12781-12788 (2011).

6 P. Poggiolini, J. Lightw. Technol. 30, 38573879 (2012).

7 P. Johannisson et al., J. Lightw. Technol. 31, 1273-1282 (2013).

8 A. Bononi et al., arXiv:1205.2193 (2013).

9 A. Vannucci et al., J. Lightw. Technol. 20, 1102-1112 (2002).

10 E. Ciaramella et al., IEEE Photon. Technol. Lett.17, 91-93 (2005). 